\title{
Dosimetric accuracy of the Acuros XB and Anisotropic analytical algorithm near interface of the different density media for the small fields of a 6- MV flattening -filter-free beam
}

\author{
S.W. Kang1, J.B. Chung2*, J.W. Lee 3 , M.J. Kim¹, Y.L. Kim4, \\ J.S.Kim², K.Y.Eom², I.A. Kim², T.S. Suh* \\ ${ }^{1}$ Department of Biomedical Engineering and Research Institute of Biomedical Engineering, College of Medicine, \\ The Catholic University of Korea, Korea \\ ${ }^{2}$ Department of Radiation Oncology, Seoul National University Bungdang Hospital, Seongnam, Korea \\ ${ }_{3}^{3}$ Department of Radiation Oncology, Kunkuk University Medical Center, Korea \\ ${ }^{4}$ Department of Radiologic Technology, Choonhae College of Health Sciences, Ulsan 689-784, Korea
}

- Original article

\author{
*Corresponding authors: \\ Dr. Jin-Beom Chung, \\ Dr. Tae. S. Suh, \\ E-mail: \\ jbchung1213@gmail.com \\ suhsanta@catholic.ac.kr \\ Revised: April 2016 \\ Accepted: June 2016 \\ Int. J. Radiat. Res., April 2017; \\ 15(2): 157-165 \\ DOI: $10.18869 /$ acadpub.ijrr.15.2.157
}

\begin{abstract}
Background: This study was conducted to assess the accuracy of dose calculation near the air-phantom interface of a heterogeneous phantom for Acuros XB (AXB) and Anisotropic Analytical Algorithm (AAA) algorithm of a 6-MV flattening-filter-free beam, compared with film measurements. Materials and Methods: A phantom included air gap was specially manufactured for this study. In order to evaluate the dose near air gapphantom interface, Eclipse treatment planning system equipped both $A X B$ and AAA was used for the dose calculations. Measurements in this region were performed with radiochromic film. The central-axis dose (CAD) and offaxis dose (OAD) between calculations and measurements were analyzed for various field sizes and air gaps. The root-mean-square-error (RMSE) was used to evaluate the difference between the calculated and measured OAD. In order to quantify agreement between the calculated and measured dose distributions, the gamma analysis was performed with the $2 \% / 2 \mathrm{~mm}$ and $3 \% / 3 \mathrm{~mm}$ criteria. Results: For all fields traveling through 1 and $3 \mathrm{~cm}$ air gap, the maximum difference in the calculated CAD was $-5.3 \%$ for AXB and $214.8 \%$ for AAA, compared to the measured CAD. For the RMSE between the calculated and measured $O A D$, the calculated $O A D$ using $A X B$ showed interval in the RMSE (from 4.4 to 12.7) while using AAA indicated broad (from 7.7 to 101.0). In addition, the gamma passing rates showed that $A X B$ was higher agreement than AAA. Conclusion: This study demonstrated that AXB was more accurate in heterogeneous media near air-phantom interface than AAA when comparing the measured data.
\end{abstract}

Keywords: Acuros XB, anisotropic analytical algorithm, heterogeneous media, FFF, interface.

\section{INTRODUCTION}

An air gap-tissue interface irradiated by high energy photon has a transition area in which the electron fluence is composed of electrons generated in both media (1). Radiation dose at proximate air cavity-tissue interfaces has been long a subject of investigation. However, to measure dose at the interface was difficult due to lack of electronic equilibrium (2). These electron disequilibrium cause an inaccurate radiation therapy. Several investigators reported that the under dosing occurs in treatment of head and neck cancer due to the presence of air 
cavities (3-7). On the other hand, the under dosing that gives rise to the skin-sparing effect could reduce the dose delivered to the rectal wall during treatment of prostate cancer, as reported by Holmes et al. (1).

Unfortunately, conventional dose calculation algorithms such as Pencil Beam Convolution Algorithm (PBC) and Anisotropic Analytical Algorithm (AAA) implemented in the current widely used treatment planning systems (TPS) cannot predict the accurate dose in the heterogeneous region including the different density materials as well as the interface between air cavities and tissues.

In recent, the Acuros XB (AXB) which called a new photon dose calculation algorithm was implemented in Eclipse TPS (Varian Medical System, Palo Alto, CA). The AXB algorithm solves deterministically the coupled system of linear Boltzmann transport equations (LBTEs) that describes the macroscopic behavior of radiation particles as they travel through and interact with matter. Some researchers stated that the AXB provides more fast and accurate in heterogeneous media as well as homogenous media than the AAA using comparisons with measurement and Monte Carlo (MC) calculations (7-10). Fogliata et al. concluded that AXB provides a valid and accurate alternative to $\mathrm{MC}$ simulations for small and large fields. In the previous study, we demonstrated that the dose calculation results using AXB on a phantom containing an air cavity was within 5\% compared to the film measurement data for small fields travelling through air cavity with a 6 -MV flattened (FF) beam (11).

In this study, we compared the accuracy of AXB and AAA against film measurements near air gap-phantom interface on heterogeneous phantom for small fields of a 6-MV flattening-filter-free (FFF) beam. To the best of our knowledge, only one paper was published the results of comparison between the calculated and measured percentage depth dose (PDD) curve but the study included not the analysis about the off-axis dose (OAD) (12). Therefore, we included the evaluation about the off-axis dose (OAD) as well as the central-axis dose (CAD). Furthermore, we analyzed the agreement between the calculated and measured dose distribution with both gamma criteria.

The aim of this study was to assess the dosimetric accuracy of AXB dose calculation near air-phantom interface of a heterogeneous phantom using a comparison with AAA and film measurements for small fields of a 6-MV FFF beam.

\section{MATERIALS AND METHODS}

\section{Phantom with an air gap}

The acryl $\left(\rho=1.14 \mathrm{~g} / \mathrm{cm}^{3}\right)$ phantom included an air gap was used to evaluate the accuracy of dose calculation for both AAA and AXB dose calculation algorithms. The overall dimensions of the heterogeneous phantom were $20 \times 20 \times$ $13.5 \mathrm{~cm}^{3}$. The phantom was composed to two types which has 1 and $3 \mathrm{~cm}$ air gap, respectively, as shown in figure 1 . The phantom was scanned using a Philips Big-bore computed tomography (CT) scanner (Philips, Healthcare Andover, MA) with a slice thickness of $3 \mathrm{~mm}$. The digital imaging and communications in medicine (DICOM) CT datasets of the phantom were then transferred to the Eclipse TPS (version 11.0.34, Varian Medical Systems, Palo Alto, CA).

\section{Calculation of the central-axis dose and off-axis dose}

By the phantom setup shown in figure 1, the central-axis dose (CAD) and off-axis dose (OAD) was calculated in interface (position A) and rebuild-up regions (position $B$ and $C$ ) beyond air gap of the phantom for four field sizes from $2 \times 2$ $\mathrm{cm}^{2}$ to $5 \times 5 \mathrm{~cm}^{2}$. The position $\mathrm{A}$ indicates the air -phantom interface. The position $\mathrm{B}$ and $\mathrm{C}$ indicate the regions of 3 and $5 \mathrm{~mm}$ depth beyond air gap within the heterogeneous phantom.

All dose computations were performed with both AAA and AXB dose calculation algorithms equipped in Eclipse TPS for a 6-MV FFF beam of TrueBeam linear accelerator (Varian Medical Systems, Palo Alto, CA, USA). A dose of $400 \mathrm{cGy}$ was delivered at maximum dose depth $(1.5 \mathrm{~cm})$ by $100 \mathrm{~cm}$ source-to-surface distance (SSD). The 
calculation grid size of $0.1 \mathrm{~cm}$ was used to improve the dose calculation accuracy.

\section{Measurement of the central axis dose and off axis dose}

Measurement of the CAD and OAD was performed on the heterogeneous phantom with radiochromic film (EBT3, the same batch, Lot \#: 3171401). The CAD, OAD, and dose distributions were measured in position $\mathrm{A}, \mathrm{B}$ and $\mathrm{C}$ under the same condition of calculations in
TPS.

For the film dosimetry, a net-optical density (netOD) curve for a 6-MV FFF beam was obtained with a $10 \times 10 \mathrm{~cm}^{2}$ field size. Doses ranging from 0 to 900 cGy were used to acquire the calibration curve of the film. After irradiated 24 hours, the films were scanned with a flatbed scanner (Epson Expression 11000 XL, Epson America Inc., Long Beach, CA) for analyzing the measured $\mathrm{CAD}, \mathrm{OAD}$, and dose distributions.

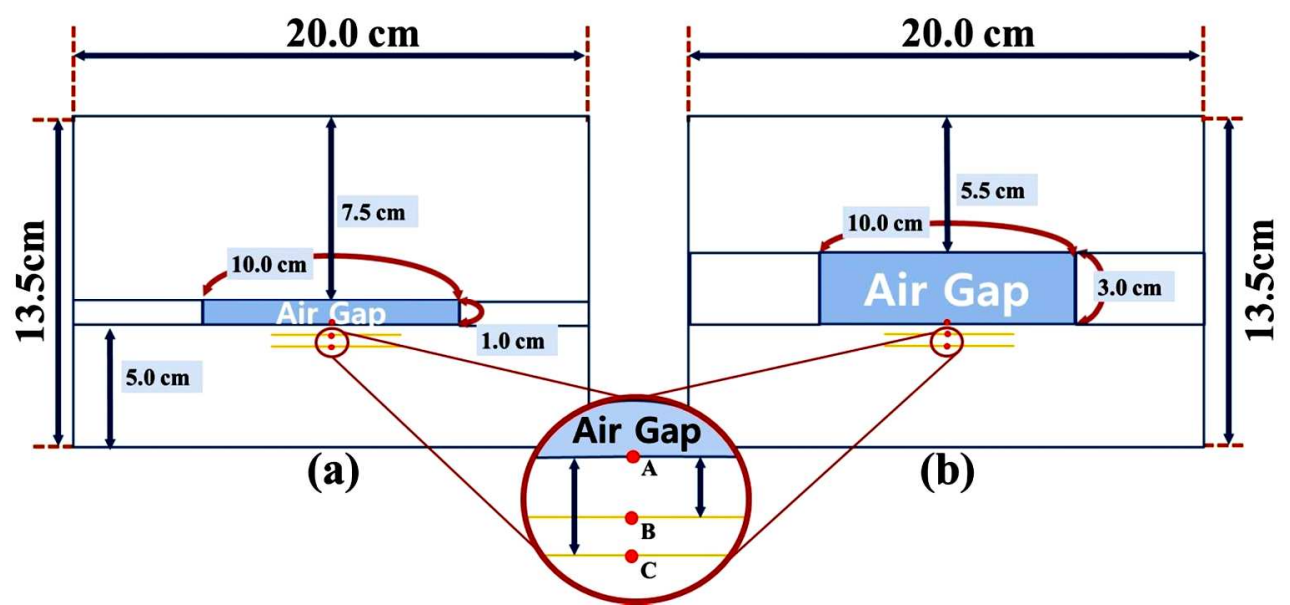

Figure 1. The inhomogeneous phantom with two air gap thickness; (a) 1 and (b) $3 \mathrm{~cm}$ gap thickness.

\section{Analysis between the calculated and measured data}

For analysis of the calculated CAD and OAD, the calculated results which were acquired in TPS were resized into the same resolution of the measured data by the interpolation method. With matching the calculated CAD, the percentage difference (\%Diff) in the calculated CAD against the measured CAD was evaluated across four field sizes and three positions. The \% Diff was expressed as the following equation (1):

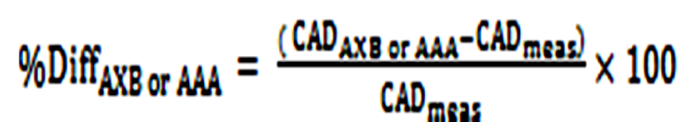

Also, the difference between the calculated and measured OAD was evaluated with the function of the root-mean-square-error (RMSE). The RMSE was defined as the following Equation (2):

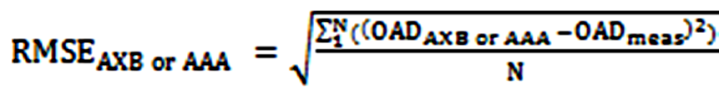

where, $\mathrm{N}$ is the total point number for the $O A D$. $O A D_{A X B}$ or AAA represents the calculated $O A D$ by $\mathrm{AXB}$ or $\mathrm{AAA}$ algorithm and $O \mathrm{AD}_{\text {meas }}$ is the measured OAD by film.

In order to quantify the agreement between the calculated and measured dose distributions, a 3-D gamma analysis was performed as described by Moran et al. (13) The gamma analysis applied the modified gamma index $\left(\Gamma_{\mathrm{m}}\right.$, Equation 3), which adds the generalized gradient $\left(\mathrm{G}_{\mathrm{e}}\right)$ to the conventional gamma equation. The $G_{e}$ (Equation 4), which combines all local gradients at the evaluated point $\left(\mathrm{r}_{\mathrm{e}}\right)$ with the nearest surrounding point $\left(\mathrm{r}_{\mathrm{j}}\right)$, is calculated from the dose difference $\left(\Delta \mathrm{d}_{\text {re-ri }}\right)$ and grid spacing $\left(\Delta \mathrm{d}_{\text {re-rij }}\right)$ between the evaluated point $\left(\mathrm{r}_{\mathrm{e}}\right)$ and the nearest point $\left(r_{j}\right)$ in the evaluated matrix (14).

Int. J. Radiat. Res., Vol. 15 No. 2, April 2017 


$$
\begin{gathered}
\Gamma_{m}\left(r_{r}, r_{e}\right)=\sqrt{\left(\frac{r\left(r_{r}, r_{e}\right.}{\Delta d_{m}}\right)^{2}+\left(\frac{\Delta D\left(r_{r}, r_{e}\right) \mid-G_{e} d_{g c}}{\Delta D_{M}}\right)^{2}} \\
r\left(r_{r}, r_{e}\right)=\left|r_{r}-r_{e}\right|, \Delta D\left(r_{r}, r_{e}\right)=D_{r}\left(r_{r}\right)-D_{e}\left(r_{e}\right),(3) \\
G_{e}=\sqrt{\sum_{j}\left(\frac{\Delta d_{r_{e}}-r_{j}}{\Delta x_{r_{e}}-r_{j}}\right)^{2}}
\end{gathered}
$$

The 3D gamma agreement was evaluated with the criteria of distance to agreement $\left(\Delta \mathrm{d}_{\mathrm{M}}\right)$ of $2 \mathrm{~mm}$ and dose difference $\left(\Delta \mathrm{D}_{\mathrm{M}}\right)$ of $2 \%(2 \% / 2$ $\mathrm{mm}$ ) and $3 \% / 3 \mathrm{~mm}$.

\section{RESULTS}

\section{The calculated and measured central axis dose}

Figure 2 shows a comparison with the measured and calculated CAD at position A, B, and $C$ for the field sizes ranging from $2 \times 2$ to $5 \times$ $5 \mathrm{~cm}^{2}$ on the phantom included 1 and $3 \mathrm{~cm}$ air gap. Table 1 presents the percentage difference between the measured and calculated CAD for four fields traversing through both air gaps. When comparing the measured CAD at the position A for all fields, the maximum percentage difference in the calculated CAD using AXB for 1 and $3 \mathrm{~cm}$ air gap was $-6.6 \%$ for the $3 \times 3 \mathrm{~cm}^{2}$ field size and $-5.3 \%$ for the $2 \times 2 \mathrm{~cm}^{2}$ field size, respectively. Whereas, the percentage difference in the calculated CAD using AAA ranged from $21.6 \%$ to $59.5 \%$ for $1 \mathrm{~cm}$ air gap and from $65.5 \%$ to $214.8 \%$ for $3 \mathrm{~cm}$ air gap, depending on various field sizes.
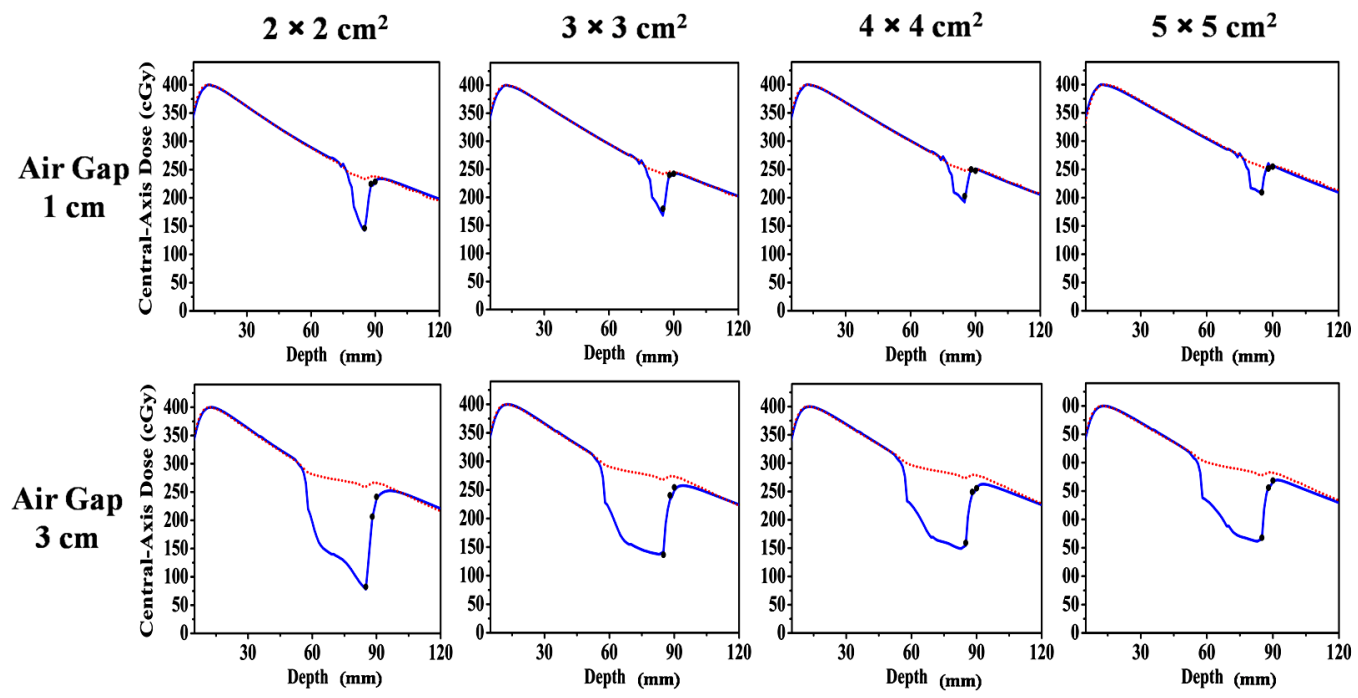

- Meas.

AXB

Figure 2. The calculated and measured central-axis dose near air-phantom interface beyond air gap of (a) 1 and (b) $3 \mathrm{~cm}$ within heterogeneous phantom for four field sizes.

In the position $\mathrm{B}$ and $\mathrm{C}$, the average percentage difference in the calculated CAD using AXB for all fields was $1.02 \%$ and $-1.16 \%$ for 1 and $3 \mathrm{~cm}$ air gap. The corresponding value in the calculated CAD using AAA was $2.1 \%$ and $12.1 \%$, respectively.

The calculated and measured off-axis dose. Figure 3 and 4 show the comparison results between the measured and calculated OAD at three positions relative to air gap and field size for both algorithms. Table 2 summarizes the Int. J. Radiat. Res., Vol. 15 No. 2, April 2017
RMSE value between the calculated and measured $\mathrm{OAD}$ at three positions depending on field size and air gap.

Compared to the measured OAD, the RMSE values in the calculated $\mathrm{OAD}$ using $\mathrm{AXB}$ were within $12.6 \mathrm{cGy}$ for $1 \mathrm{~cm}$ air gap, whereas corresponding values in the calculated CAD using AAA were more than $53.8 \mathrm{cGy}$. Overall, the RMSE value in the calculated OAD using AXB at the position $A$ was higher than that of $B$ and $C$ position for the same field size, except at the $2 \times$ 
$2 \mathrm{~cm}^{2}$ field size (RMSE value at A position: 4.4 cGy, at B and C position: 6.5 and 7.4 cGy, respectively). The RMSE values in $3 \mathrm{~cm}$ air gap were higher than those in $1 \mathrm{~cm}$ air gap for both algorithms. The maximum RMSE value in the calculated OAD using AXB and AAA compared to the measured OAD was 14.5 and 101.0 cGy. It was observed at the position A of the $3 \times 3 \mathrm{~cm}^{2}$ field size for $\mathrm{AXB}$ and the $5 \times 5 \mathrm{~cm}^{2}$ field size for
AAA.

As shown in figure 3 and 4, the relatively high dose difference between the calculated and measured OAD was obtained in the out-of-field than in-field. The difference for both algorithms was increased with increasing field size and air gap compared to the measured OAD. Furthermore, the difference was reduced with increasing measurement depth (position C).

Figure 2. The calculated and measured central-axis dose near air-phantom interface beyond air gap of (a) 1 and (b) $3 \mathrm{~cm}$ within heterogeneous phantom for four field sizes.

\begin{tabular}{|c|c|c|c|c|c|c|c|c|c|c|c|}
\hline & & \multicolumn{6}{|c|}{ Dose (cGy) } & \multicolumn{4}{|c|}{ \%Diff } \\
\hline & \multirow[b]{2}{*}{ Position } & \multicolumn{3}{|c|}{$1 \mathrm{~cm}$ air gap } & \multicolumn{3}{|c|}{$3 \mathrm{~cm}$ air gap } & \multicolumn{2}{|c|}{$1 \mathrm{~cm}$ air gap } & \multicolumn{2}{|c|}{$3 \mathrm{~cm}$ air gap } \\
\hline & & Meas. & $A X B$ & AAA & meas & $A X B$ & AAA & $A X B$ & AAA & $A X B$ & AAA \\
\hline \multirow{3}{*}{$2 \times 2 \mathrm{~cm}^{2}$} & $A^{*}$ & 146.4 & 142.5 & 233.6 & 82.4 & 78.0 & 259.3 & -2.7 & 59.5 & -5.3 & 214.8 \\
\hline & $\mathrm{B}^{*}$ & 224.6 & 222.7 & 237.8 & 206.9 & 203.3 & 266.2 & -0.8 & 5.9 & -1.7 & 28.7 \\
\hline & $\mathrm{C}^{*}$ & 228.6 & 229.9 & 237.6 & 242.0 & 237.3 & 266.1 & 0.6 & 3.9 & -2.0 & 10.0 \\
\hline \multirow{3}{*}{$3 \times 3 \mathrm{~cm}^{2}$} & $A$ & 179.7 & 167.9 & 242.0 & 136.6 & 142.2 & 268.4 & -6.6 & 34.6 & 4.1 & 96.6 \\
\hline & $\mathrm{B}$ & 239.8 & 242.7 & 245.6 & 240.1 & 233.1 & 274.1 & 1.2 & 2.4 & -2.9 & 14.2 \\
\hline & $\mathrm{C}$ & 242.0 & 243.2 & 244.9 & 254.5 & 249.1 & 273.4 & 0.5 & 1.2 & -2.1 & 7.4 \\
\hline \multirow{3}{*}{$4 \times 4 \mathrm{~cm}^{2}$} & $A$ & 202.8 & 192.2 & 248.3 & 159.3 & 154.6 & 274.1 & -5.2 & 22.5 & -2.9 & 72.1 \\
\hline & $\mathrm{B}$ & 250.0 & 252.9 & 251.2 & 249.1 & 245.7 & 279.1 & 1.2 & 0.5 & -1.4 & 12.1 \\
\hline & C & 247.6 & 251.0 & 250.5 & 255.1 & 256.9 & 278.5 & 1.4 & 1.2 & 0.7 & 9.2 \\
\hline \multirow{3}{*}{$5 \times 5 \mathrm{~cm}^{2}$} & $A$ & 209.4 & 207.8 & 254.6 & 167.9 & 165.6 & 277.9 & -0.8 & 21.6 & -1.4 & 65.5 \\
\hline & $B$ & 251.3 & 260.6 & 254.4 & 256.0 & 258.6 & 282.7 & 3.7 & 1.2 & 1.0 & 10.4 \\
\hline & $C$ & 255.0 & 256.3 & 255.7 & 269.1 & 266.7 & 282.2 & 0.5 & 0.3 & -0.9 & 4.9 \\
\hline
\end{tabular}

${ }^{*} A$ : air-phantom distal interface, $B$ and $C: 3$ and $5 \mathrm{~mm}$ depths below air cavity.

(a)
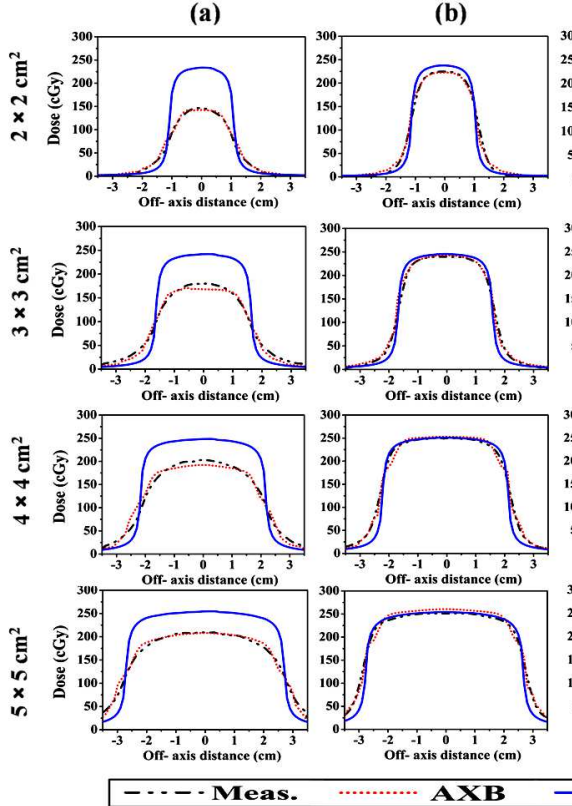

AXB

Figure 3. Comparison between the measured and calculated off-axis dose at each position for all fields traversing through a (c)
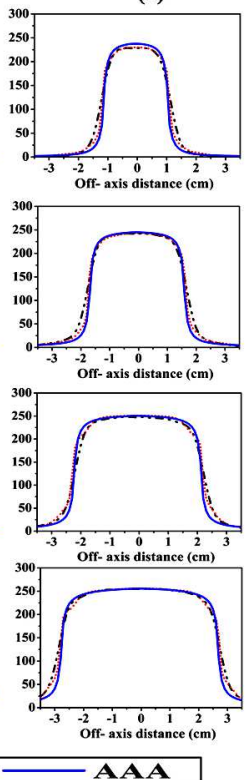
$1 \mathrm{~cm}$ air gap. (a)
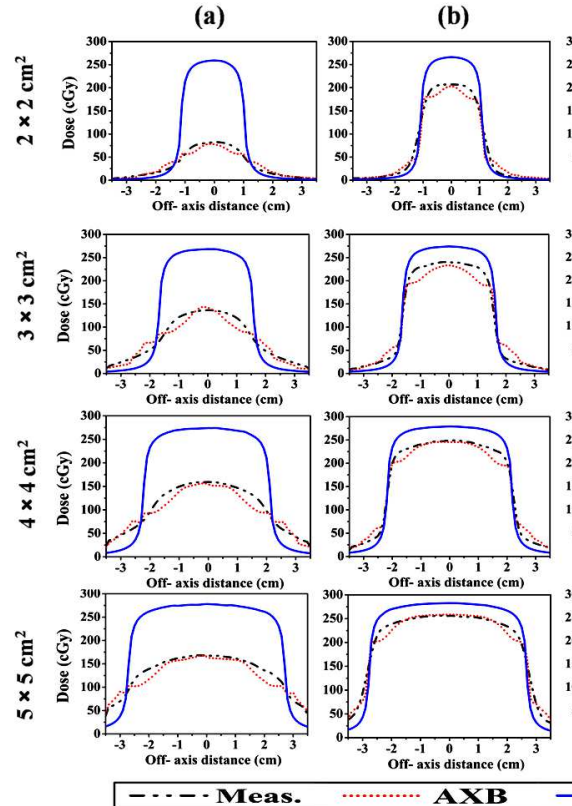

AXB

Figure 4. Comparison between the measured and calculated off-axis dose at each position for all fields traversing through a $3 \mathrm{~cm}$ air gap.

Int. J. Radiat. Res., Vol. 15 No. 2, April 2017 
Kang et al. / Accuracy of Acuros XB and Anisotropic algorithm

\begin{tabular}{|c|c|c|c|c|c|c|}
\hline & & \multicolumn{2}{|c|}{ 1 cm air gap } & \multicolumn{2}{|c|}{$3 \mathbf{c m}$ air gap } \\
\hline Field size & & AXB & AAA & & AXB & AAA \\
\hline \multirow{3}{*}{$2 \times 2 \mathrm{~cm}^{2}$} & A $^{*}$ & 4.4 & 51.2 & 6.6 & 93.7 \\
\cline { 2 - 6 } & B $^{*}$ & 6.5 & 16.5 & & 12.3 & 33.7 \\
\cline { 2 - 6 } & $\mathrm{C}^{*}$ & 7.4 & 9.1 & & 10.9 & 16.7 \\
\hline \multirow{3}{*}{$3 \times 3 \mathrm{~cm}^{2}$} & A & 10.1 & 53.8 & 14.5 & 91.9 \\
\cline { 2 - 6 } & B & 7.2 & 15.7 & 11.5 & 28.6 \\
\cline { 2 - 6 } & C & 6.4 & 8.5 & 10.9 & 16.5 \\
\hline \multirow{3}{*}{$4 \times 4 \mathrm{~cm}^{2}$} & A & 12.6 & 52.5 & 12.7 & 96.3 \\
\cline { 2 - 6 } & B & 7.4 & 17.9 & 12.4 & 30.6 \\
\hline \multirow{3}{*}{$5 \times 5 \mathrm{~cm}^{2}$} & C & 8.4 & 8.4 & 10.0 & 21.4 \\
\cline { 2 - 6 } & A & 9.3 & 51.8 & 11.8 & 101.0 \\
\cline { 2 - 6 } & C & 9.3 & 7.7 & 7.7 & 11.4 & 31.5 \\
\hline
\end{tabular}

${ }^{*} \mathrm{~A}$ : air-phantom distal interface, $\mathrm{B}$ and $\mathrm{C}: 3$ and $5 \mathrm{~mm}$ depths below air cavity.

Table 3. Gamma passing rate between the calculated and measured dose distributions evaluated with $2 \% / 2 \mathrm{~mm}$ and $3 \% / 3 \mathrm{~mm}$ criteria at all selected positions for all fields traversing though 1 and $3 \mathrm{~cm}$ air gap.

\begin{tabular}{|c|c|c|c|c|c|c|c|c|c|}
\hline \multirow[b]{3}{*}{$\begin{array}{l}\text { Field } \\
\text { size }\end{array}$} & & \multicolumn{4}{|c|}{$1 \mathrm{~cm}$ air gap } & \multicolumn{4}{|c|}{$3 \mathrm{~cm}$ air gap } \\
\hline & & \multicolumn{2}{|c|}{ AXB } & \multicolumn{2}{|c|}{ AAA } & \multicolumn{2}{|c|}{ AXB } & \multicolumn{2}{|c|}{ AAA } \\
\hline & & $2 \% / 2 \mathrm{~mm}$ & $3 \% / 3 \mathrm{~mm}$ & $2 \% / 2 \mathrm{~mm}$ & $\begin{array}{c}3 \\
3 \% / \mathrm{mm}\end{array}$ & $2 \% / 2 \mathrm{~mm}$ & $3 \% / 3 \mathrm{~mm}$ & $2 \% / 2 \mathrm{~mm}$ & $3 \% / 3 \mathrm{~mm}$ \\
\hline \multirow{3}{*}{$\begin{array}{c}2 \times 2 \\
\mathrm{~cm}^{2}\end{array}$} & $A^{*}$ & 75.6 & 99.1 & 4.1 & 12.1 & 62.5 & 90.0 & 2.5 & 13.6 \\
\hline & $\mathrm{B}^{*}$ & 80.6 & 98.2 & 18.8 & 44.7 & 71.2 & 91.9 & 17.2 & 31.5 \\
\hline & $\mathrm{C}^{*}$ & 90.0 & 99.5 & 66.9 & 83.7 & 86.7 & 93.9 & 23.8 & 48.1 \\
\hline \multirow{3}{*}{$\begin{array}{c}3 \times 3 \\
\mathrm{~cm}^{2}\end{array}$} & $\mathrm{~A}$ & 78.0 & 97.5 & 4.1 & 6.1 & 61.4 & 94.2 & 2.7 & 11.8 \\
\hline & $B$ & 84.2 & 98.6 & 70.6 & 81.2 & 76.0 & 97.7 & 11.9 & 30.1 \\
\hline & $\mathrm{C}$ & 94.1 & 98.8 & 81.2 & 87.8 & 94.8 & 99.5 & 18.8 & 38.4 \\
\hline \multirow{3}{*}{$\begin{array}{c}4 \times 4 \\
\mathrm{~cm}^{2}\end{array}$} & $A$ & 77.2 & 99.5 & 2.5 & 5.6 & 76.9 & 86.4 & 5.1 & 10.3 \\
\hline & $B$ & 85.1 & 99.7 & 80.0 & 86.7 & 82.9 & 97.6 & 9.9 & 22.0 \\
\hline & C & 94.8 & 99.6 & 84.7 & 91.5 & 96.7 & 99.6 & 11.5 & 23.7 \\
\hline \multirow{3}{*}{$\begin{array}{c}5 \times 5 \\
\mathrm{~cm}^{2}\end{array}$} & $A$ & 80.4 & 99.3 & 3.8 & 6.0 & 80.1 & 93.2 & 0.2 & 3.0 \\
\hline & $B$ & 87.0 & 100.0 & 87.8 & 98.5 & 87.2 & 98.5 & 7.2 & 17.5 \\
\hline & $\mathrm{C}$ & 99.0 & 100.0 & 93.6 & 97.7 & 98.3 & 99.2 & 9.5 & 34.9 \\
\hline
\end{tabular}

*A: air-phantom interface, B and C: 3 and $5 \mathrm{~mm}$ depth below air gap within heterogeneous phantom.

\section{Gamma evaluation}

Figure 5 and table 3 show agreement between the calculated and measured dose distribution. The calculated dose distribution using AXB was better agreement than that of AAA. As shown in Table 3, the passing rate for all fields and air gaps indicated higher agreement in the calculated dose distribution using AXB than AAA.

For all fields traversing through a $1 \mathrm{~cm}$ air gap, the passing rates for $\mathrm{AXB}$ ranged from $75.6 \%$ to $99.0 \%$ for a criterion of $2 \% / 2 \mathrm{~mm}$, whereas the corresponding values for AAA Int. J. Radiat. Res., Vol. 15 No. 2, April 2017 ranged from $4.1 \%$ to $93.6 \%$. The passing rate for $\mathrm{AXB}$ and AAA was increased as increasing the measured depth as shown in table 3 . In the position $\mathrm{A}$, the maximum passing rate between the calculated and measured dose distribution was $80.4 \%$ for $\mathrm{AXB}$ and $4.1 \%$ for AAA. In the position $\mathrm{C}$, the passing rate was $99.0 \%$ for $\mathrm{AXB}$ and $93.6 \%$ for AAA.

For both algorithms, the passing rates were decreased with increasing air gap. In a $3 \mathrm{~cm}$ air gap, the maximum passing rates of the calculated dose distribution using AXB and AAA was $98.3 \%$ and $23.8 \%$ for the $2 \% / 2 \mathrm{~mm}$ criteria. 


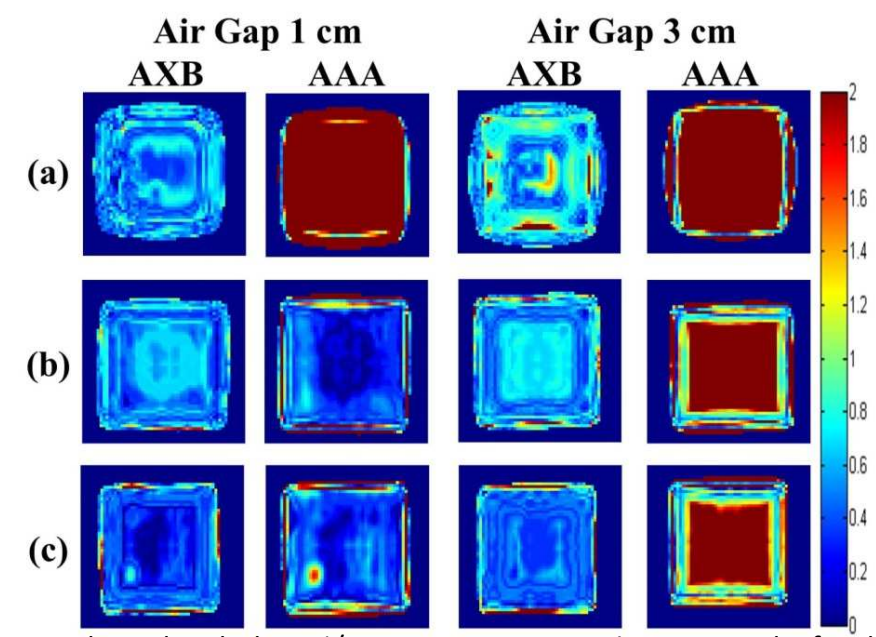

Figure 5. Gamma distribution evaluated with the $3 \% / 3 \mathrm{~mm}$ criteria at position $\mathrm{A}, \mathrm{B}$, and $\mathrm{C}$ for the $4 \times 4 \mathrm{~cm} 2$ field traversing though 1 and $3 \mathrm{~cm}$ air gap. The color bar indicates the gamma-index levels.

The gamma passing rate at the position $\mathrm{C}$ was significantly decreased in the calculated dose distribution using AAA than AXB.

In all positions $(A, B$, and $C)$, the passing rates of the calculated dose distribution using AXB for the $3 \% / 3 \mathrm{~mm}$ criteria were more than $90.0 \%$ for two air gaps and all fields tested, excepted $86.4 \%$ at $A$ position for the $4 \times 4 \mathrm{~cm}^{2}$ field. The passing rates of the calculated dose distribution using AXB for the $2 \% / 2 \mathrm{~mm}$ criteria were mostly closed than $80 \%$. On the other hand, the passing rates of the calculated dose distribution using AAA for all positions showed a wide range (from 5.6 to 98.5 for a $1 \mathrm{~cm}$ air gap and from 3.0 to 48.1 for a $3 \mathrm{~cm}$ air gap) depending on the field size, and air gap.

\section{DISCUSSION}

Many studies reported on the accuracy verification of the different dose calculation algorithms to heterogeneous media as well as homogeneous medium (18-22). Some studies demonstrated that the AXB was more accurate with measurement in heterogeneous phantom than AAA ${ }^{(5,18,19)}$. Kan et al. ${ }^{(18)}$ also indicated that the differences of AXB was within $7.5 \%$ at the different material interface of the heterogeneous phantom compared to TLD measurement for clinical IMRT, whereas those of the AAA overestimated more than $11.0 \%$. With clinical aspect, Chung et al. (20) presented that the AXB was more accurate than the AAA at dose calculation in lung stereotactic ablative radiotherapy (SABR).

We also evaluated the accuracy on the calculated dose of $\mathrm{AXB}$ and $\mathrm{AAA}$ respect to film measurement. The verification for both algorithms was performed as a function of field size and air gap with a phantom included air gap, specially using a 6-MV FFF beam. To our knowledge, no study has compared the dosimetric accuracy between the different dose calculation algorithms and measurements for FFF beams.

Our results demonstrated that AXB was lower difference than AAA in the calculated CAD and OAD compared to the measured data (table 1 ). For the position $A, B$, and $C$, the calculated CAD using AXB was observed the differences of the narrow (within $-6.6 \%$ for $1 \mathrm{~cm}$ air gap and -5.3 for $3 \mathrm{~cm}$ air gap) interval with the measured CAD as a function of field size, while AAA has the relatively (up to $214.8 \%$ for $3 \mathrm{~cm}$ air gap) broad differences. In results from the previous two studies, the AXB was the difference of the calculated CAD within $7.3 \%$ on a phantom included an air gap against the measured CAD. (18) Rana et al., also reported that the differences in CAD between AXB calculations and measurements ranged from $-3.81 \%$ to $0.9 \%$ across the several air gap thickness $(2,4$, and 6 $\mathrm{cm})$ for three field sizes $(3 \times 3,5 \times 5$, and $10 \times 10$

Int. J. Radiat. Res., Vol. 15 No. 2, April 2017 
$\mathrm{cm}^{2}$ ) using a 6-MV photon beam. However, there was no the regular trend for the difference between the calculated and measured CAD as changing the field size and air gap. Our results are in line with this finding.

In RMSE used to evaluate the calculated and measured OAD, the maximum value in RMSE relative to the field size and air gap was 12.7 for AXB and 101.0 cGy for AAA, respectively. It presented in the position $A$ which was air-phantom interface adjacent the different densities. The RMSE value of both algorithms was decreased with increasing filed size and reducing air gap thickness compared to the measurement. The remarkable difference was observed in the out-of-field of profile for the AAA than the AXB, as shown figures 3 and 4. The discrepancy was explained that AAA did not model the effect of lateral electronic disequilibrium. For inhomogeneity correction in the heterogeneous media, the AAA was considered with two parameters which were independently scaled depth-directed and lateral components. The depth-directed component was scaled by calculating radiologic distance between the surface and the point of interest, whereas the lateral component calculated the path length from the central of the beamlet in the water equivalent environment. The divergent scatter of heterogeneities from the upper levels was incorporated correctly. In contrast, the AXB is able to calculate the interactions of radiation particle within the heterogeneity media by using LBTE. Therefore, the AXB can perform dose calculations with the material assignment for each voxel distinguished by the Hounsfield unit (HU) of the CT image $(23,24)$.

In this study, gamma evaluation was performed to compare agreement between the calculated and measured dose distributions. This gamma analysis has been widely accepted and provides the means for an efficient analysis. Our study indicated that the AXB calculations were good agreement with the measured results than AAA for both criteria in spite of variations of the field size and air gap (figure 5 and table 3). For three selected positions, the highest passing rate in both algorithms was observed in position $\mathrm{C}$ which had the deepest depth. The lowest passing rate was shown in interface (position A) as in CAD and RMSE. In the previous study reported by Kan et al.. (25), there was also low gamma passing rates in the air-tissue interface.

In this study, all measurements in our heterogeneous phantom were made with radiochromic EBT3 film. Therefore, the results were obtained at depth which included the film thickness. They were not exactly the doses at air -phantom interface although the differences between calculations and measurements were not large. Moreover, the film dosimetry had a disadvantage which requires the well-controlled calibration procedure. However, it was a well-estimated method for verifying both absolute dose and dose distribution.

\section{CONCLUSION}

With using a phantom including air gaps, the accuracy of dose calculation algorithm of AAA and AXB was evaluated with film measurement for small fields and three positions near air-phantom interface. Our study found that the AXB was more accurate dose calculation algorithm than the AAA compared to the measured dose according to variations of field size and air gap when considering a heterogeneous media, especially included air gap. Therefore, the results of this study suggest that the AXB must be used in heterogeneous media such as human body where include significantly different densities for achieving accurate dose calculation.

\section{ACKNOWLEDGEMENTS}

This research was supported by a grant of the Korea Health technology R\&D Project through the Korea Health Industry Development Institute (KHIDI), funded by the Ministry of Health \& Welfare, Republic of Korea. (Grant Number: HI15C0638) and by a grant number (2014R1A2A1A10050270) from the Mid-career Researcher Program through the National 
Research Foundation of Korea funded by the Ministry of Science, ICT \& Future Planning.

\section{Conflicts of interest: Declared none.}

\section{REFERENCES}

1. Li XA, Yu C, Holmes T (2000) A systematic evaluation of air cavity dose perturbation in megavoltage X-ray beams. Med Phys, 27: 1011-1017.

2. Klein EE, Chin LM, Rice RK, Mijnheer BJ (1993) The influence of air cavity on interface doses for photon beams. Int J Radiat Oncol Biol Phys, 27: 419-427.

3. Kan WK, Wu PM, Leung HT, Lo TC, Chung CW, Kwong DL, Sham ST (1998) The effect of nasopharyngeal air cavity on x-ray interface doses. Phys Med Biol, 43: 529-537.

4. Ostwald PM, Kron T, Hamilton CS (1996) Assessment of mucosal underdosing in larynx irradiation. Int $J$ Radiat Oncol Biol Phys, 36: 181-187.

5. Kan WK Leung HT, So RWK, Yu PKN (2013) Experimental verification of the Acuros $X B$ and $A A A$ dose calculation adjacent to heterogeneous media for IMRT and RapidArc of nasopharygeal carcinoma. Med Phys, 40: 1-18.

6. Solberg TD, Holly FE, Salles AAF, Wallace RE, Smathers JB (1995) Implications of tissue heterogeneity for radiosurgery in head and neck tumors. Int I Radiat Oncol Biol Phys, 32: 235-239.

7. Kan WK, Leung HT, Yu KN (2013) Dosimetric impact of using the Acuros XB algorithm for intensity modulated radiation therapy and RapidArc planning in nasophagryngeal carcinomas. Int J Radiat Oncol Biol Phys, 85: 73-80.

8. Han T, Mikell JK, Salepour M, Mourtada F (2011) Dosimetric comparison of Acuros $\mathrm{XB}$ deterministic radiation transport method with Monte Calro and model-based convolution methods in heterogeneous media. Med Phys, 38: 2651-2664.

9. Fogliata A, Nicolini G, Clivio A, Vanetti E, Mancosu P, Cozzi $L$ (2011) Dosimetric validation of the Acuros XB advanced dose calculation algorithm: Fundamental characterization in water. Phys Med Biol, 56: 1879-1904.

10. Bush K, Gane IM, Zavgorodni S, Ansbacher W, Beckham W (2011) Dosimetric validation of Acuros XB with Monte Calro for photon dose calculations. Med Phys, 38: 22082221.

11. Koo T, Chung JB, Eom KY(2015), Dosimetric effects of the acuros XB and anisotropic analytical algorithm on volumetric modulated arc therapy planning for prostate cancer using an endorectal balloon. Radiation Oncology, 10: 48 .

12. Rana S and Rogers K (2013) Dosimetric evaluation of Acuros $X B$ dose calculation algorithm with measurements in predicting doses beyond different air gap thickness for smaller and larger filed sizes. J Med Phys, 38: 9-14.

13. Jean MM, Jeffrey R, Benedick AF (2004) A dose-gradient analysis tool for IMRT QA. Journal of Applied Clinical Medical Physics, 6: 62-73.

14. Park JY, Lee JW, Choi KS, Lee JS, Kim YH, Hong S, Suh TS (2011) Development of a novel quality assurance system based on rolled-up and rolled-out radiochromic films in voumetric modulated arc therapy. Med Phys, 38: 66886696.

15. Vassiliev O, Wareing T, McGhee J, Failla G. Salehpour MR, Mourtada $F$ (2010) Validation of a new grid based Blotzmann equation solver for dose calculation in radiotherapy with photon beams. Phys Med Biol, 55: 581-598.

16. Fogliata A, Nicolini G, Clivio A, Vanetti E, Cozzi L (2011) Dosimetric evaluation of Acuros XB Advanced Dose Calculation algorithm in heterogeneous media. Radiat Oncol, 6: 82-97.

17. Bush K, Gagne S, Zavgorodni WA, Beckham W (2011) Dosimetric validation of Acuros XB with Monte Carlo methods for photon dose calculations. Med Phys, 38: 2208-2221.

18. Kan MWK, Leung LHT, Yu PKN (2012) Verification and dosimetric impact of Acuros XB algorithm on intensity modulated stereotactic radiotherapy for locally persistent nasopharyngeal carcinoma. Med Phys, 39: 4705-4714.

19. Kang SW, Suh TS, Chung JB ,Eom KY, Kim IA ,Kim JS, Lee JW, Park JY (2015) Dosimetric Accuracy of AAA and Acuros $X B$ Dose Calculations within an Air Cavity for Small Fields of a 6-MV Flattening Filter-free Beam. Journal of the Korean Physical Society, 67: 2138-2145.

20. Chung JB, Eom KY, Kim IA, Kim JS, Lee JW, Hong S, Kim YL, Park BM, Kang SW, Suh TS (2014) Comparison of Anisotropic Analytic Algorithm Plan and Acuros XB Plan for Lung Stereotactic Ablative Radiotherapy Using Flattening FilterFree Beams. PROGRESS in MEDICAL PHYSICS, 25: 210-217.

21. Han T, Followill D, Mikell J, Repchak R, Molineu A, Howell R, Salehpour M, Mourtada F (2013) Dosimetric impact of Acuros $\mathrm{XB}$ deterministic radiation transport algorithm for heterogeneous dose calculation in lung cancer. Med Phys, 40: 1-11.

22. Han T, Mourtada F, Kisling K, Mikell J, Followill D, Howell R (2012) Experimental validation of deterministic Acuros XB algorithm for IMRT and VMAT dose calculations with the Radiological Physics Center's head and neck phantom. Med Phys, 39: 2193-2202.

23. Han T, Mikell J, Salehpour M, Mourtada F (2011) Dosimetric comparison of Acuros XB deterministic radiation transport method with Monte Carlo and model-based convolution methods in heterogeneous media. Med Phys, 38: 2651-2664.

24. Mark RA, Christine HS, Jeffrey S, Cox L, Mohan R (2000) The impact of electron transport on the accuracy of computed dose. Med Phys, 27: 1266-1274.

25. Kan MWK, Cheung JYC, Leung LHT, Yu PKN (2011) The accuracy of dose calculations by anisotropic analytical algorithms for stereotactic radiotherapy in nasopharyngeal carcinoma. Phys Med Biol, 56: 397-413. 
